\title{
Quando o responder antigo torna-se um comportamento novo: Descrição de um processo de mudança clínica ${ }^{1}$
}

\author{
When the old responding turns into a new behavior: Describing a clinical change process \\ Francielly Perón ${ }^{[a]}$, Jocelaine Martins da Silveira ${ }^{[b]}$
}

\footnotetext{
${ }^{[a]}$ Mestranda em Psicologia pela Universidade Federal do Paraná (UFPR), professora adjunta do Departamento de Psicologia da Universidade Federal do Paraná (UFPR), doutora em Psicologia Clínica pela Universidade Federal do Paraná (UFPR), Curitiba, PR - Brasil, e-mail: jocelainesilveira@ufpr.br

${ }^{[b]}$ Doutora em Psicologia Clínica pela Universidade de São Paulo (USP), professora adjunta no Departamento de Psicologia da Universidade Federal do Paraná (UFPR), Curitiba, PR - Brasil.
}

Recebido: 09/07/2010 Received: 07/09/2010

Aprovado: 27/10/2010 Approved: 10/27/2010

\section{Resumo}

Supondo que a mudança clínica está estreitamente relacionada ao tipo de classe que é enfraquecida ou fortalecida, conforme a sistematização feita pela Psicoterapia Analítica Funcional (FAP), recorreram-se aos conceitos de Modelagem, Diferenciação e Indução para analisar e discutir um processo de mudança comportamental na clínica. 0 objetivo do estudo foi descrever um processo de mudança clínica em que a classe de resposta considerada problema transforma-se em uma classe de resposta relacionada à melhora. 0 método consistiu em analisar 15 sessões iniciais realizadas com uma cliente atendida em uma clínica-escola pública. Os resultados expõem quatro dimensões identificadas, as quais foram sendo diferenciadas ao longo do processo terapêutico. Discutiu-se o processo de mudança clínica, considerando dimensões do responder que sofreram diferenciação do longo da terapia.

Palavras-chave: Análise comportamental clínica. Psicoterapia Analítica Funcional (FAP). Mudança clínica.

\begin{abstract}
The main assumption of this study was that clinical change is related to a response class that lacks strength while another gains it, according to the Functional Analytic Psychotherapy (FAP). The concepts of Modeling, Differentiation and Induction were used to analyse and to discuss a clinical change process. The aim of this study was to describe a clinical change process in which the so-called problematic response turns into a type of response related to improvement. Clinical relevant behaviors (CRB) were registered in protocols, filed in during each session by the therapist and by a trained observer. Then, CRB was examined in order to identify the dimensions that were being selected within sessions until a new operant class had been installed. The results show four dimensions that were being differentiated during the therapy. The clinical change process was discussed, considering the dimensions of response that suffered differentiation and the relevance of the client/therapist relationship in such change.
\end{abstract}

Keywords: Clinical Behavior Analysis. Functional Analytic Psychotherapy (FAP). Clinical change.

${ }^{1} \mathrm{O}$ estudo contou com o fomento financeiro do Tesouro Nacional, na forma de bolsa de iniciação científica para a primeira autora. 
A aprendizagem que acontece no contexto clínico envolve um tipo específico de mudança comportamental. Trata-se de uma diminuição da frequência de certos comportamentos e do aumento na frequência de outros. Tais frequências relacionam-se com o sofrimento do cliente e as alterações nelas serão chamadas no presente artigo de 'mudança clínica'. A mudança comportamental é um fenômeno controverso (Shahan \& Chase, 2002; Skinner, 1935) e sua compreensão no contexto clínico tem um importante valor para o campo aplicado (Gottman \& Rushe, 1993).

Catania (1998/1999, p. 137) define uma classe de respostas como aquela cujas respostas compartilham consequências comuns, isto é, produzem o mesmo efeito no ambiente. "O fato é que é mais importante definir as classes de comportamentos por suas consequências do que por suas topografias".

De acordo com Catania (1998/1999, p. 411), a capacidade de responder de maneiras novas é possível graças ao processo de modelagem, definida como uma "modificação gradual de alguma propriedade do responder (frequentemente, mas não necessariamente, a topografia) pelo reforço diferencial de aproximações sucessivas a uma classe operante alvo".

Skinner (1953/2003) compara o processo de modelagem com o trabalho de um escultor. Segundo Skinner, o comportamento operante é o produto final de um processo de modelagem no qual os pontos que se sucedem não são inteiramente distintos.

O condicionamento operante modela o comportamento como o escultor modela a argila. Ainda que algumas vezes o escultor pareça ter produzido um objeto inteiramente novo, é sempre possível seguir o processo retroativamente até a massa original indiferenciada e fazer que os estágios sucessivos, por meio dos quais retornamos a essa condição sejam tão pequenos quanto quisermos. Em nenhum ponto emerge algo que seja muito diferente do que o precedeu. 0 produto final parece ter uma especial unidade ou integridade de planejamento, mas não se pode encontrar o ponto em que ela repentinamente apareça. No mesmo sentido, um operante não é algo que surja totalmente desenvolvido no comportamento do organismo. É o resultado de um contínuo processo de modelagem (Skinner, 1979/2003, p. 101).

Segundo Catania (1998/1999), a variabilidade é a propriedade que torna a modelagem efetiva. Uma população de respostas é necessária para a seleção de um determinado conjunto delas. 0 reforço diferencial envolve quatro diferentes processos: indução, generalização, diferenciação e discriminação, (Catania, 1998/1999).

A classe de resposta é modelada pelo reforço diferencial, que é caracterizado como o reforçamento apenas das respostas desejáveis, o que resulta em um processo de 'diferenciação'. Eventualmente, respostas muito próximas àquelas desejáveis continuam sendo emitidas, mesmo não sendo reforçadas. Elas são chamadas de respostas de 'indução' e são consideradas por alguns, sinônimo de generalização, como, por exemplo, Skinner (1979/2003) as concebe.

Segundo Dinsmoor (2004), Skinner empregou o termo 'indução' para designar a expansão do efeito do reforço entre respostas similares ao mesmo estímulo. Ele havia sido influenciado pela terminologia usada por Pavlov (1927), que se referiu a mudanças na magnitude da resposta salivar ao estímulo condicional que eram acompanhadas por mudanças similares na mesma resposta a outro estímulo. Pavlov chamou esse fenômeno de generalização.

Assim, Dinsmoor (2004) afirma que Pavlov não precisou nomear a expansão correspondente ao efeito do reforço entre respostas similares ao mesmo estímulo. Mas Skinner precisou nomear a expansão do efeito do reforço de acordo com o grau de similaridade entre diferentes formas do responder. Então, ele designou o fenômeno de 'indução', qualificando-a de indução entre estímulos ou entre respostas.

Skinner (1953/2003, p. 147) exemplifica o fenômeno da indução quando respostas emocionais são eliciadas diante de pessoas estranhas que se parecem com conhecidos. Define indução como: "[...] um termo que descreve o fato de que o controle adquirido por um estímulo é compartilhado por outros estímulos com propriedades comuns".

0 termo 'indução' é empregado por Skinner (1979/2003, p. 147) com muita proximidade à acepção de 'generalização'. Diz o autor: "A indução (ou generalização) não é uma atividade do organismo [...]". Já Catania (1998/1999) estabelece uma distinção clara afirmando que, enquanto a indução diz respeito à expansão das respostas para outros contextos; a generalização está relacionada com a expansão dos estímulos. De acordo com Catania (1998/1999), o processo de indução caracteriza-se pelo fenômeno da expansão do reforço a uma dada resposta para respostas com propriedades similares, não incluídas na classe reforçada. 
Skinner (1979/2003, p. 105) distingue os processos de reforço operante e de reforço diferencial: "No primeiro caso, estamos interessados em como o comportamento é adquirido, no segundo, em como é refinado. É a diferença entre 'saber como se faz alguma coisa' e "fazê-la bem". 0 último é o campo da habilidade".

Assim, o reforço operante é uma operação entre o responder e o ambiente, que reforça as respostas que tendem a aumentar a probabilidade de ocorrência deste responder, enquanto que o reforço diferencial reforça apenas as respostas com propriedades pré-selecionadas. 0 reforçador operante pode ser positivo, quando sua apresentação aumenta a probabilidade de resposta ou o reforçador operante pode ser negativo quando a sua remoção aumenta a probabilidade de resposta (Catania, 1998/1999).

Quando o reforço diferencial é programado para as propriedades da resposta, ele resulta nos processos de 'indução' e de 'diferenciação', por outro lado, se for programado para as propriedades dos estímulos, resultará nos processos de 'generalização' e 'discriminação' (Catania, 1998/1999).

Catania (1998/1999, p. 138) esclarece que a resposta pode variar quanto a qualquer dimensão de resposta. "O reforço diferencial pode ser baseado em qualquer dimensão de resposta, de modo que qualquer dimensão ou combinação de dimensões pode fornecer as propriedades definidoras de uma classe operante". 0 autor chama de dimensões: o lo$\mathrm{cal}$, a força, a topografia e a direção. E segundo ele, a Psicologia da Aprendizagem dá ênfase em três dimensões, estudadas ao longo da história, a saber:

1) organização temporal do comportamento;

2) padrão sequencial;

3) variabilidade comportamental e a novidade.

O reforço diferencial pode atuar na organização temporal nas propriedades de latência, de duração e de ritmo. Catania (1998/1999) evidencia a separação de respostas no tempo, para principalmente, esclarecer que o reforço diferencial pode aumentar ou diminuir a taxa de respostas em um período de tempo. Descaracterizando, com isso, a obrigatoriedade da observação da taxa de respostas no processo do reforço, uma vez que pode ser reforçada de forma diferencial uma resposta somente se precedida de um tempo mínimo sem uma resposta. 0 autor ilustra com a situação de um carro afogado, no qual o motorista deve aguardar um determinado período de tempo sem dar partida para que o carro volte a ligar. Dessa forma, considera-se que a taxa de respostas é apenas mais uma propriedade do comportamento que pode ser diferenciada dentro da dimensão da organização temporal.

Ao descrever uma sucessão de comportamentos, há duas possíveis análises, cada uma de acordo com a natureza da ordenação sequencial: há o encadeamento de respostas, na qual a resposta reforça a antecedente e discrimina a consequente; como também há respostas independentes uma das outras, apenas que se apresentam na ordem apropriada (Catania, 1998/1999).

Quando é observado um comportamento original, o responder pode não se encaixar na classe de respostas que está sendo reforçada, porém há propriedades neste novo comportamento que podem ser definidas como uma classe operante. É importante perceber que esses novos comportamentos só foram possíveis porque houve outras respostas reforçadas anteriormente. Catania (1998/1999) ilustra com o responder de um golfinho de saltar para fora da água com um giro em espiral. Para o autor, esse responder só foi possível após o processo de modelagem, ou seja, após serem reforçados, por exemplo, comportamentos de saltar para trás, bater na água com a cauda e pular na borda da piscina.

Catania $(1998 / 1999$, p. 144$)$ alerta que os organismos estão sujeitos a várias respostas e a várias consequências ao longo do tempo, deve-se, portanto evitar, analisar as sequências individuais resposta-estímulo. 0 autor esclarece: "[...], o problema é identificar a dimensão por meio da qual o responder pode passar a se conformar à classe de respostas que tem consequências". Contudo, percebe-se que o novo responder pode ser mantido por uma consequência diferente daquela reforçada anteriormente, mas que é o resultado de um processo de diferenciação.

Delimitou-se conceitualmente, até o momento, 'reforço diferencial', 'classe de resposta', 'indução', 'generalização', 'discriminação' e 'diferenciação'. Tais noções são importantes para a compreensão da Psicoterapia Analítica Funcional (FAP), uma estratégia terapêutica, fundamentada no Behaviorismo Radical. A FAP sistematiza os fenômenos comportamentais do contexto clínico em três classes de resposta do cliente. Os comportamentos classificados como CRB1 são aqueles relacionados ao problema e devem ter a sua frequência diminuída ao longo da 
terapia. Normalmente, os CRB1 são interpretados como fuga/esquiva de estímulos aversivos presentes em uma relação interpessoal. As respostas classificadas em CRB2 são consideradas os progressos do cliente, e que devem ter a frequência aumentada ao longo das sessões. Elas ocorrem em baixa frequência no início da terapia e concorrem com a classe de CRB1. Uma terceira classe de respostas é chamada de CRB3 e diz respeito às interpretações dos clientes acerca dos próprios problemas (Kohlenberg \& Tsai, 1991/2001). Diversos estudos têm discutido o efeito das intervenções terapêuticas e supervisões clínicas que empregam a FAP (Beckert, 2002; Brandão \& Silveira, 2004; Bush, Kanter, Callaghan, Baruck, Weeks \& Berlin, 2009; Callaghan, 2006a, b; Callaghan, Summer \& Weidman, 2003; Conte \& Brandão, 2001; Kanter, Landes, Busch, Rush, Brown \& Baruk, 2006; Kanter, Schildcrout \& Kohlenberg, 2005; Vandenberghe, 1999, 2008; Vandenberghe \& Ferro, 2005). A rigor, adotando a conceituação proposta por Kohlenberg e Tsai (1991), a expressão CRB aplica-se a instâncias do comportamento clinicamente relevante que ocorrem durante uma sessão de terapia. Adotou-se aqui, entretanto, uma compreensão mais ampla, segundo a qual, um comportamento se traduz por uma classe de respostas, as quais podem ocorrer em contextos diversos (dentro ou fora da sessão). Atualmente, outras expressões têm sido adotadas para designar essas especificidades como 1 (ocorrência do comportamento problema fora da sessão) e; 2 (ocorrência fora da sessão do comportamento concorrente ao problema) (Callaghan, Follette, Ruckstuhl \& Linnerooth, 2008).

Assim, ao longo do tratamento, presume-se que o reforço diferencial transforme uma classe de CRB1 predominante, em uma classe de CRB2, por meio da indução e diferenciação do responder. Isso leva a supor que as dimensões do responder em cada classe são fundamentais para a modelagem que o terapeuta implementará. De um ponto de vista operacional, a estratégia da FAP permite a identificação dos processos de modelagem, de indução, de diferenciação, de reforço diferencial, bem como a classificação dos responderes em classe de respostas.

Dessa forma, buscou-se, na presente pesquisa, responder à questão: como o responder adquire novas propriedades e passa a ser mantido por uma nova consequência ao longo de um processo terapêutico? Isto é, de que modo uma classe de CRB1, transforma-se, na interação terapeuta/cliente em uma classe de CRB2?

0 presente estudo se propôs a investigar o processo de diferenciação de respostas em uma situação de mudança comportamental no contexto clínico. Assim, o objetivo do presente estudo foi descrever um processo de mudança clínica comportamental em que se empregou a estratégia da FAP nos atendimentos, identificando as dimensões da classe de respostas considerada problema (CRB1), que passam a ser diferenciadas e induzidas até tornarem-se dimensões de uma classe de respostas relacionada à melhora (CRB2).

\section{Método}

\section{Dados da terapeuta}

A terapeuta foi uma estudante que atendia pela primeira vez como participante de uma outra pesquisa (que tinha por objetivo avaliar o efeito de um programa de treino da FAP nos relatos de CRB feito por terapeutas em treino ainda não iniciados nessa estratégia terapêutica). Na ocasião, a terapeuta cursava o sexto período do curso de Psicologia na Universidade Federal do Paraná.

\section{Dados da cliente}

A cliente tinha 23 anos, era professora e estudante do terceiro grau. Era solteira durante o processo terapêutico, começou a namorar Arthur (nome fictício), um colega estudante.

\section{Local do atendimento}

Os atendimentos ocorreram na clínica-escola do curso de Psicologia da Universidade Federal do Paraná. Utilizou-se para os atendimentos uma sala com espelho unidirecional.

\section{Queixa}

A cliente apresentou uma queixa de ansiedade "generalizada" no dia a dia. Relatou sentir-se ansiosa diante de novos desafios, principalmente em seu trabalho, como professora em escolas de idiomas. Relatou: Eu tive vários problemas por causa da minha 
ansiedade, inclusive de saúde. A minha ansiedade tem me atrapalhado nos meus empregos, nos meus relacionamentos. Eu sou assim, tenho até uma certa tendência a falar e a andar rápido. E isso tudo, às vezes atrapalha (sic). Acrescentou que chega a sabotar seu próprio trabalho a fim de provocar sua demissão. Relatou que apresentava comportamento inadequado para uma professora, como por exemplo: ausentar-se das aulas várias vezes para ir ao banheiro; ausentar-se da aula para tomar água; e atender ao telefone celular durante o período de aula. A vida afetiva com rapazes era considerada pela cliente como "conturbada", tendo relatado dificuldades nos três últimos relacionamentos. A cliente relata ter sido agressiva naquelas interações afetivas.

\section{Análise de contingências}

Após avaliação do caso, em uma formulação em termos de CRB, considerou-se que a cliente apresentava uma classe de resposta problema que pareceu ser mantida por supressão, adiamento ou anulação, de sofrer rejeição por alguém com quem tivesse estabelecido contato íntimo e comprometido. Essa classe foi considerada o CRB1. E a classe relacionada à melhora clínica, ou seja, a classe de CRB2, uma classe concorrente com o CRB1, seria a de respostas mantidas pela consequência de atenção e carinho do outro - respostas passíveis de alguma rejeição interpessoal.

Os relatos da cliente acerca do próprio comportamento fora da sessão terapêutica sugeriam fortemente a consequenciação de suspender, adiar ou anular o contato íntimo e comprometido com o outro que redundaria em algum risco de rejeição/ humilhação. No início do tratamento, a classe de respostas CRB1 foi interpretada em diversos âmbitos da vida pessoal da cliente. São exemplos de interações típicas dessa classe:

a) Com os amigos: Eu tinha marcado com a minha amiga pra sair com ela. Mas ela desmarcou em cima da hora... Odeio quando ela faz isso. Vou dar um gelo nela (sic);

b) Com a família: Na minha casa é normal. Não gosto muito de contar as coisas para a minha mãe porque ela se mete muito na minha vida (sic);

c) Nos relacionamentos amorosos: Eu acho o Arthur meio chato. Eu gosto de pessoas que dão emoção na minha vida (sic); d) No trabalho: Odeio que se metam no meu trabalho. Se eu precisar, eu peço conselho. Por isso ganhei fama de barraquenta nos meus empregos (sic).

Da mesma forma, apesar de infrequentes, alguns comportamentos relatados pela cliente fora da sessão terapêutica foram interpretados como CRB2, ou seja, como comportamentos conservados pela consequência comum de manter a atenção ou o carinho do outro, arriscando-se a alguma forma de rejeição. Os exemplos a seguir ilustram relatos ainda no início do tratamento:

a) Nos relacionamentos amorosos: Eu briguei com o Arthur. Chorei muito. Depois falei que eu tenho que aprender a falar as coisas de outra maneira... (sic);

b) Na família: Eu contei pra minha mãe que a minha menstruação estava atrasada. Ela me perguntou se eu estava grávida (sic).

\section{Procedimento}

Primeiramente, foi feito o contato com a cliente, a qual autorizou a realização da pesquisa, assinando o Termo de Consentimento Livre e Esclarecido. A escolha da participante foi realizada a partir do prontuário de triagem, que reunia duas informações essenciais para a sua participação na pesquisa: a queixa, estreitamente ligada a dificuldades interpessoais e o horário disponível para frequentar a terapia, o mesmo disponível pela terapeuta e pela observadora.

Depois da escolha da participante, foram realizadas 15 sessões de terapia registradas em videoteipes e em um protocolo de registro de CRB (a cliente teve alta terapêutica no fim de 2007, totalizando 42 sessões realizadas). Esse protocolo de registros era preenchido pela terapeuta e por um observador, treinado em identificação de CRBs, que permaneceu na sala de espelhos unidirecional. Em um formato de quadro, o protocolo relacionava em cinco colunas aspectos referentes aos CRBs emitidos pela cliente durante a sessão: o número de CRB observado; o intervalo de tempo em que foi emitido (dividido em frações de cinco minutos); se o CRB ocorreu fora ou dentro da sessão; a descrição da resposta classificada em CRB; e a classificação desse CRB em 1, 2 ou 3. Contou-se um CRB a cada consequenciação feita pelo terapeuta ou a cada mudança de comportamento da cliente. 


\section{Análise dos dados}

A análise dos dados foi realizada a partir das duas fontes de registro: o protocolo de registros de CRBs e os videoteipes. 0 registro das sessões em videoteipe foi usado para a transdução de descrições de cada sessão e para o levantamento de dimensões dentro de cada CRB que foram sendo selecionadas ao longo das sessões. Os resultados obtidos das ses-

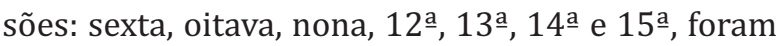
analisados apenas dos protocolos de registro, uma vez que os videoteipes em que foram realizadas as filmagens estavam danificados. Houve danos em alguns videoteipes, sendo utilizadas para a análise dos dados dessas sessões apenas as fichas de registro da terapeuta e da observadora. As filmagens da nona, $14^{\underline{a}}$ e $15^{\underline{a}}$ sessões foram danificadas integralmente e as filmagens da sexta, oitava, $12^{\underline{a}}$ e $13^{\underline{a}}$ sessões, registraram tempo parcial da sessão, a saber, respectivamente: 14 minutos, 24 minutos, 45 minutos e 30 minutos.

Quando houve divergência entre os números de CRBs registrados no protocolo de registros e no videoteipe, optou-se por considerar aquele registro que tivesse o maior número de CRBs.

Posteriormente, cada CRB identificado possibilitou a descrição de quatro itens: os eventos antecedentes à resposta da cliente; a resposta da cliente típica do CRB; a dimensão crítica diferenciada durante a terapia e que era observável naquele responder; e os eventos consequentes promovidos na própria interação com a terapeuta.

A partir dessas descrições, foram verificadas quatro principais dimensões selecionadas a partir dos CRB1 e CRB2 ao longo da terapia, a saber: falar sobre algo; manter-se ao tema proposto pela terapeuta; falar de si e de sentimentos; valorizar o outro. A partir do levantamento destas principais dimensões selecionadas, foram montados os histogramas apresentados na seção de Resultados.

\section{Dimensões selecionadas ao longo do processo terapêutico}

As principais dimensões que foram selecionadas ao longo do processo terapêutico foram: falar sobre algo; manter-se ao tema proposto pela terapeuta; falar de si e de sentimentos; e valorizar o outro. A dimensão 'falar sobre algo' foi evidenciada principalmente em comportamentos de fuga/esquiva da cliente de verbalizar acontecimentos pessoais e em comportamentos de fuga/esquiva de manter-se a temas aversivos. A dimensão 'manter-se ao tema proposto pela terapeuta' diz respeito aos comportamentos da cliente que foram consistentes com as deixas verbais fornecidas pela terapeuta. A dimensão 'falar de si e de sentimentos' refere-se às verbalizações da cliente acerca de si mesma. E a dimensão 'valorizar o outro' relaciona-se a componentes da verbalização da cliente em que havia valorização de alguma coisa ou de alguém. Muitas vezes, por se tratar de comportamentos emitidos dentro das sessões, essa dimensão era identificada na valorização da terapia ou da terapeuta.

A Tabela 1 apresenta o modelo da sistematização das respostas da cliente, interpretadas como o CRB1 e o CRB2, relacionadas com as dimensões críticas diferenciadas ao longo da terapia e os eventos antecedentes e consequentes na interação com a terapeuta.

Tabela 1 - Classe de Respostas da Cliente, interpretada, respectivamente, na primeira e na segunda linhas, como CRB1 e CRB2, relacionadas com as dimensões críticas diferenciadas ao longo da terapia e os eventos antecedentes e consequentes na interação com a terapeuta

\begin{tabular}{|c|c|c|c|}
\hline Eventos antecedentes & Resposta da Cliente & $\begin{array}{l}\text { Dimensões críticas diferenciadas } \\
\text { ao longo da terapia }\end{array}$ & $\begin{array}{l}\text { Eventos consequentes } \\
\text { promovidos pela terapeuta }\end{array}$ \\
\hline $\begin{array}{l}\text { Terapeuta apresentou dica para } \\
\text { a cliente relatar a sua queixa. }\end{array}$ & $\begin{array}{l}\text { Relatou que prefere } \\
\text { a linha da Psicanálise } \\
\text { porque não gosta de } \\
\text { ouvir conselhos. }\end{array}$ & $\begin{array}{l}\text { Falou sobre algo, apresentando inte- } \\
\text { resse em trabalhar na terapia (apesar } \\
\text { de ter valorizado outra abordagem } \\
\text { clínica). }\end{array}$ & Acenou com nutos positivos. \\
\hline $\begin{array}{l}\text { Terapeuta resumiu o que foi } \\
\text { relatado pela cliente na sessão. }\end{array}$ & $\begin{array}{l}\text { Relatou estar melhor } \\
\text { depois de ter falado na } \\
\text { terapia. }\end{array}$ & Sugeriu estar valorizando a terapia. & $\begin{array}{l}\text { Comentou ter ficado feliz com } \\
\text { o comentário. }\end{array}$ \\
\hline
\end{tabular}

Fonte: Dados da pesquisa. 


\section{Resultados}

Os resultados do presente estudo estão relacionados com as dimensões que, ao longo da terapia, sofreram o processo de diferenciação até que uma nova classe de respostas pudesse ser notada. Estão dispostos na seguinte ordem: respostas que compõem o CRB1 da cliente; respostas que compõem o CRB2 da cliente; explicitação das principais dimensões selecionadas ao longo do processo terapêutico; a apresentação dos resultados quantitativos obtidos (CRB1, CRB2 e dimensões selecionadas) reunidos em figuras, a fim de possibilitar uma compreensão do processo de modelagem.

A Figura 1 apresenta a frequência de CRB1 e de CRB2 ao longo das sessões.

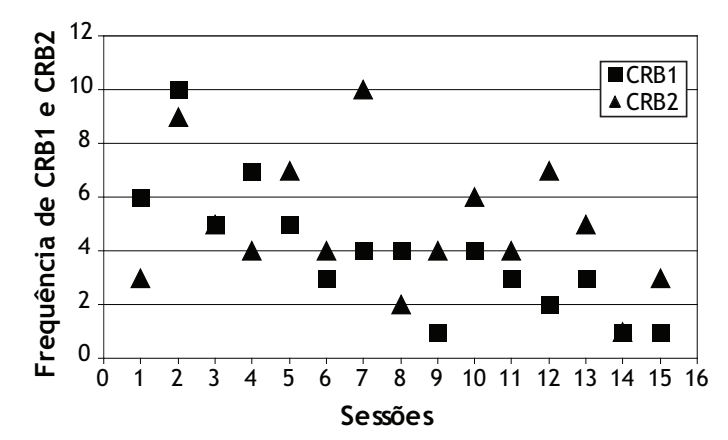

Figura 1 - Frequência da classe de respostas CRB1 e CRB2 ao longo das sessões

Fonte: Dados da pesquisa.

A Figura 1 indica que ao término das 15 sessões foram totalizados 59 CRB1s emitidos pela cliente dentro da sessão terapêutica. Destaca-se um declínio na frequência de comportamentos ao longo do processo terapêutico, uma vez que até a quinta sessão foram registrados 33 CRB1s acumulados, enquanto que nas últimas dez sessões foram registradas 26 CRB1s acumulados. Na primeira sessão, foram observados seis CRB1s, enquanto, na 15a sessão, apenas um CRB1 foi registrado. Se comparados esses dados, caracterizariam uma redução de aproximadamente $83,4 \%$ na frequência de CRB1s registrados na primeira e na $15^{\text {a }}$ sessão.

A Figura 1 indica 74 CRB2s emitidos pela cliente dentro da sessão terapêutica ao longo das 15 sessões. A sessão em que mais foram registrados
CRB2s, foi a sétima, na qual constaram 10 CRB2s. $\mathrm{Na} 14^{\mathrm{a}}$, apenas um CRB2 foi registrado.

Apesar de não haver variação no número de CRB2 registrados na primeira e na $15^{\underline{a}}$ sessões, a quantidade de CRB2 foi maior do que o registrado na primeira sessão em 11 das 15 sessões analisadas; correspondendo a um número maior de CRB2 do que o observado na primeira sessão em mais de $70 \%$ da terapia.

0 reforço diferencial oriundo da interação com a terapeuta, que incidia sobre o CRB1 foi direcionado às seguintes dimensões: (1) falar sobre algo, mesmo que caracterize comportamento de fuga/esquiva de algum tema; (2) manter-se ao tema proposto pela terapeuta, mesmo que de um modo arrogante; (3) falar sobre si e sobre seus sentimentos, mesmo com tom de superioridade; e (4) valorizar o outro, mesmo de um jeito pedante.

A Figura 2 ilustra as quatro dimensões que sofreram diferenciação a partir dos CRB1 registrados ao longo do processo terapêutico.

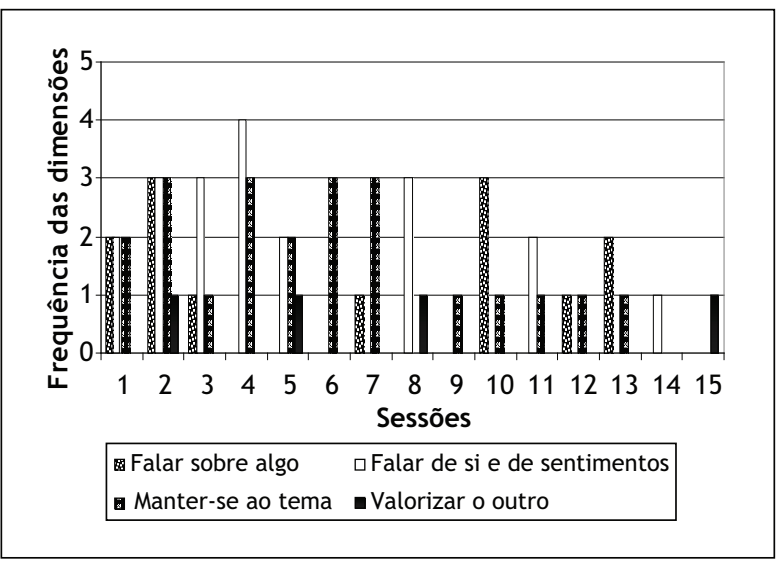

Figura 2 - Dimensões selecionadas ao longo do processo terapêutico por meio de CRB1

Fonte: Dados da pesquisa.

A Figura 2 indica que a dimensão 'manter-se ao tema proposto pela terapeuta', que pode ser interpretada como uma forma de aceitação da condução da sessão pela terapeuta constitui-se na dimensão mais continuamente selecionada - categorizada em 12 , das 15 sessões realizadas. Isso representa $80 \%$ das sessões. A referida dimensão foi selecionada em 23 dos 59 CRB1s registrados, 38,9\% do total das dimensões selecionadas. 
A dimensão 'falar sobre algo', relacionada ao comportamento de fuga/esquiva da cliente, representou $1 / 3$ das dimensões selecionadas na primeira sessão da terapia. Essa mesma dimensão não foi observada nas seguintes sessões subsequentes: quarta, quinta, sexta, oitava, nona, $11^{a}, 14^{a}$ e $15^{a}$.

Já a dimensão 'falar de si e de sentimentos' e a dimensão 'valorizar o outro', provavelmente mais susceptíveis à consequenciação por aproximar-se do outro, constituem mais de $40 \%$ das dimensões selecionadas ao longo do tratamento, integrando os CRB1 emitidos e totalizando 24 CRB1s nos quais foram diferenciadas.

A Figura 3 ilustra as mesmas quatro dimensões selecionadas ao longo do processo terapêutico integrando o CRB2.

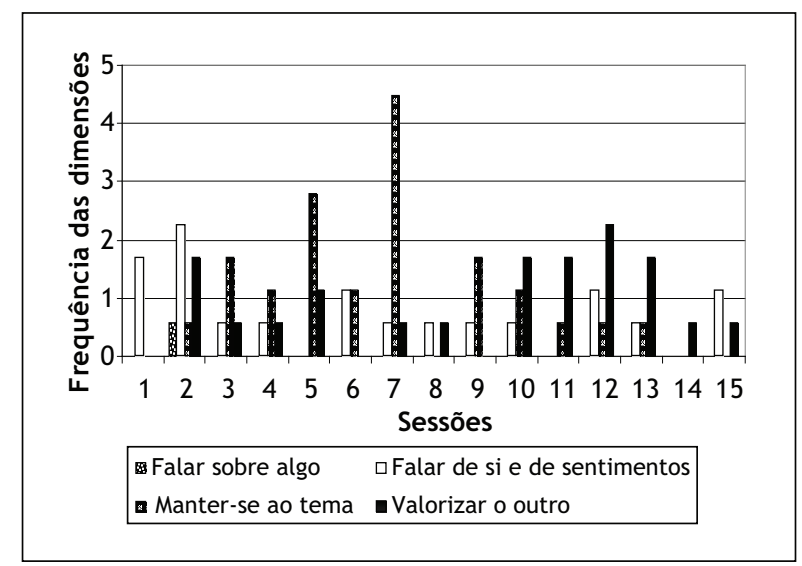

Figura 3 - Dimensões selecionadas ao longo do processo terapêutico em respostas do CRB2

Fonte: Dados da pesquisa.

A Figura 3 ilustra que as dimensões selecionadas estão na seguinte proporção: manter-se ao tema proposto pela terapeuta, ocorreu mais vezes que a de valorizar a terapia e a terapeuta, que, a seu turno, foi mais frequente que falar de si e de sentimentos, que foi mais observado do que o falar sobre algo. A dimensão 'manter-se ao tema proposto pela terapeuta' é 1,20 vezes mais frequente do que a dimensão 'valorizar a terapia e a terapeuta'; 1,45 vezes mais frequente do que a dimensão 'falar de si e de sentimentos'; e 29 vezes mais frequente do que a dimensão 'falar sobre algo'.

As dimensões 'valorizar a terapia e a terapeuta' e 'falar de si e de sentimentos', relacionadas à aproximação mais íntima com o outro, aumentaram ao longo do processo terapêutico, sendo diferenciadas a partir dos CRB2s registrados em $80 \%$ das sessões.

Com relação à dimensão falar sobre algo, relacionada a comportamentos de fuga/esquiva de uma dada temática, foi diferenciada apenas uma vez nos comportamentos de CRB2, o que corresponde a menos de $1 \%$ das dimensões selecionadas em respostas CRB2.

\section{Discussão}

A base de investigação da FAP, sugerida por Kohlenberg e Tsai (1991/2001) foi mantida, já que se observou, dentro da sessão terapêutica: (1) o reforço; (2) a especificação de CRB; e a (3) generalização.

O reforço dos comportamentos desejáveis pode ser observado em vários momentos, e em diversos procedimentos da terapeuta. Será descrito posteriormente, por estar intimamente relacionado com a elucidação do processo de modelagem por meio do reforço diferencial, questão central do presente estudo.

0 efeito da indução, descrito por Kohlenberg e Tsai (1991/2001) como generalização, relaciona-se ao procedimento do reforço diferencial e não a qualquer aspecto do reforço. 0 reforço, segundo Catania (1998/1999), fundamenta-se na probabilidade de ocorrência de uma resposta. No contexto da sessão terapêutica, o procedimento não reforça qualquer instância do comportamento do cliente. Conforme mencionado, Skinner (1953/2003) relaciona o reforço com a aquisição de um dado comportamento, mas não com seu refinamento.

Como proposto por Kohlenberg e Tsai (1991/ 2001), a observação das respostas emitidas pela cliente foi fundamental para que se pudesse especificar e classificar as classes de CRB1, CRB2 e CRB3. No presente trabalho, optou-se por examinar os CRB1s e CRB2s por tratarem-se, respectivamente, dos comportamentos problemas a serem extintos do repertório comportamental da cliente e os comportamentos desejáveis a serem incluídos no repertório comportamental da cliente ao longo da terapia, por meio do processo de modelagem. Observou-se, ao fim das 15 sessões, que as respostas interpretadas como CRB1 diminuíram e as respostas interpretadas como CRB2 aumentaram; conforme previram Conte e Brandão (2001) como resultado de uma intervenção com base na FAP. 
Observa-se o processo de indução não apenas a partir dos relatos de melhora no âmbito da vida pessoal da cliente, mas também nota-se que, no decorrer das sessões, a cliente verbaliza menos situações de conflitos e de desentendimentos. Ao longo da terapia, a cliente emitiu respostas que foram interpretadas como aproximação mais íntima com a terapeuta ao mesmo tempo em que apresentou respostas que foram interpretadas como uma aproximação mais íntima dela com os pais, com os colegas de trabalho e com próprio namorado, o que sugere um processo de indução. Supõe-se, portanto, que a mudança clínica do CRB1 em CRB2 relaciona-se fortemente com um processo de indução de dimensões observadas inicialmente dentro da classe do CRB1, conforme o aumento delas e do CRB2 apresentados nos resultados.

A modelagem realizada pelo terapeuta dirige-se a uma classe de respostas, já que uma resposta única nunca é emitida exatamente da mesma forma duas vezes (Catania, 1998/1999). Assim, a análise da modelagem foi realizada a partir da reunião desses comportamentos mantidos pela mesma consequência ambiental.

O processo de modelagem dentro da sessão terapêutica foi o resultado do reforço diferencial dispensado pela terapeuta. Segundo Catania (1998/1999, p. 411), a modelagem é uma "modificação gradual de alguma propriedade do responder (frequentemente, mas não necessariamente, a topografia) pelo reforço diferencial de aproximações sucessivas a uma classe operante alvo".

A modelagem descrita no presente estudo ilustra o processo gradual, que ocorreu em uma escala determinada de tempo - 15 sessões, de 50 minutos cada, em um intervalo de aproximadamente quatro meses. Nota-se, na décima 15ª , que o CRB1 ainda ocorria. Para a discussão da modelagem do comportamento desejado foram consideradas principalmente três hipóteses.

A primeira hipótese diz respeito à própria análise dos dados. Por terem sido danificados integralmente os videoteipes da $14^{a}$ e e $15^{a}$ a sessões, sendo utilizados para análise apenas os protocolos de registro de CRB da observadora e da terapeuta, alguns comportamentos emitidos pela cliente podem não ter sido observados e registrados. Considerando os videoteipes principalmente dessas sessões, os comportamentos classificados como CRB1 e CRB2 poderiam estar melhor descritos.
A segunda hipótese está relacionada com a inexperiência da terapeuta, que pode ter deixado de consequenciar adequadamente o comportamento da cliente. Sobretudo da primeira a quinta sessões, quando ainda não havia sido treinada na estratégia da FAP (por causa do delineamento de outro estudo, que usou os mesmos registros). E a terceira hipótese da razão porque ainda são observados CRB1s na 15a sessão, considera o número reduzido de sessões disponíveis para análise, já que o processo de modelagem é gradual e lento.

Contudo, foi possível descrever o processo de modelagem que ocorreu ao longo da terapia. Os resultados indicam que houve instauração de novos comportamentos observáveis no repertório da cliente. Conforme destacou Skinner (1953/2003), a probabilidade de um novo comportamento ocorrer ao acaso é praticamente zero, sendo necessário o processo de modelagem. Os histogramas comparativos das dimensões selecionadas ilustram novidade nas dimensões, principalmente na dimensão 'valorizar o outro'. Embora essa dimensão pareça surgir repentinamente, observa-se que é resultado do reforço diferencial a outras dimensões previamente selecionadas.

As dimensões do responder que foram selecionadas ao longo da terapia foram fundamentalmente: 'falar sobre alguma coisa'; 'manter-se ao tema proposto pela terapeuta'; 'falar de si e de sentimentos'; e 'valorizar o outro'. Ao selecionar essas dimensões e propriedades do responder da cliente, foi possível o processo de modelagem, que transformou uma classe de CRB1 em outra, e CRB2, mantida pelo contato íntimo com o outro confiável e confortável.

De acordo com Catania (1998/1999), não necessariamente, mas frequentemente, o responder se modifica inicialmente em aspectos topográficos. A funcionalidade do comportamento foi o foco do presente estudo, por preocupar-se com a identificação das novas consequências que manteriam as dimensões destacadas. Porém, esse processo só pôde ser descrito utilizando como base a topografia do responder da cliente.

0 reforço diferencial foi utilizado pela terapeuta para selecionar as dimensões que redundariam em um novo comportamento, que seria interpretado como CRB2. Assim, em todos os comportamentos emitidos pela cliente e observáveis dentro da sessão terapêutica, o reforço diferencial selecionou, ao longo da terapia, as dimensões já descritas. 
Interpretaram-se os vários eventos consequentes promovidos pela terapeuta como eventos que selecionavam certas dimensões do responder da cliente. Quando a terapeuta apenas ouvia os comentários da cliente, seja dela mesma ou de outros assuntos, interpretou-se que isso teve função de evento reforçador operando sobre o falar na terapia. Quando a terapeuta concordou com algumas afirmações verbalmente ou acenando com a cabeça, também foram sendo selecionadas as dimensões desejadas. A terapeuta relata seus próprios sentimentos e impressões, funcionado como modelo e selecionando as dimensões de ouvir e valorizar o outro.

E por fim, todo esse processo de modelagem foi realizado por meio de reforço diferencial de aproximações sucessivas a uma classe operante alvo (Catania, 1998/1999). Definiu-se como classe operante alvo aquela interpretada como CRB2 e o reforço diferencial foi utilizado com aproximações sucessivas às dimensões do responder dessa classe operante alvo.

Catania (1998/1999), ao esclarecer sobre a observação de um comportamento original, enfatiza que, apesar desse novo responder esteja sendo mantido por outras consequências, é resultado do processo de diferenciação. Dessa forma, considerou-se que, ao longo da terapia, os comportamentos emitidos pela cliente e interpretados como CRB2 estão sendo mantidos com consequências diferentes daquelas que mantêm os comportamentos interpretados como CRB1.

Percebe-se, ao fim, que essa nova classe de respostas é o resultado do processo de modelagem de uma outra classe de respostas, altamente improvável por ser concorrente. E os resultados apontaram que é possível modelar os comportamentos desejáveis a partir dos comportamentos considerados problemas.

Os resultados sugerem que o CRB1 transforma-se em uma nova classe, o CRB2, conforme as dimensões foram sendo selecionadas ao longo do processo terapêutico. A diminuição na frequência de CRB1 foi sensível a uma inspeção visual dos gráficos. Com relação à acentuação menos manifesta na curva de comportamentos CRB2, supõe-se que a forma de registrar tenha influenciado. A cada consequenciação feita pelo terapeuta ou a cada mudança de comportamento da cliente, foi registrado um CRB, de modo que há a possibilidade de um comportamento adequado ter ocorrido por períodos mais longos de tempo no decorrer da terapia e terem sido registrados como apenas uma resposta emitida. Por exemplo, um CRB2 pode ter durado vários minutos.

A dimensão 'falar sobre algo' pareceu relacionada à consequência de evitação de intimidade (que poderia redundar em rejeição). Essa dimensão foi desaparecendo durante o processo de modelagem. As dimensões consideradas mais próximas da consequência de manter a atenção e carinho do outro são: "falar de si e de sentimentos" e "valorizar o outro". A dimensão "falar de si e de sentimentos" foi sendo cada vez mais selecionada a partir dos comportamentos de CRB2. Já a dimensão "valorizar o outro" é pouco observada em comportamentos de CRB1, porém é o resultado do próprio processo de modelagem - é a dimensão que mais permite a aproximação íntima e comprometida com o outro. A dimensão "manter-se ao tema proposto", embora aparentemente pouco importante, foi a mais frequentemente selecionada ao longo do processo e dependeu um tanto quanto da apresentação de eventos antecedentes verbais por parte da terapeuta.

Enfim, as quatro dimensões analisadas no presente estudo levam a supor que o terapeuta tenha realizado fundamentalmente uma modelagem sobre o comportamento verbal da cliente. Aparentemente, mudanças no comportamento verbal da cliente são indicativos importantes de mudanças em seus comportamentos interpessoais, já que o comportamento verbal parte do comportamento social.

Estudos futuros podem avaliar o mesmo processo de reforço diferencial em combinação com procedimentos de bloqueio de fuga/esquiva sobre a classe de CRB1. Talvez, o processo terapêutico aqui analisado tenha se centrado apenas no reforço diferencial de certas dimensões do responder da cliente. Como teria sido a mudança clínica se a esse procedimento se somasse o de confrontação e bloqueio do CRB1?

\section{Conclusão}

0 presente estudo analisou um processo de mudança clínica por meio do reforço diferencial de quatro dimensões categorizadas a partir das classes de CRB1 e CRB2. Observa-se, ao longo das 15 sessões, que uma nova classe se instala, sendo então mantida por novas consequências na interação da cliente com os outros. A modelagem observada no 
presente estudo incidiu, predominantemente, sobre o comportamento verbal da cliente, o qual parece ter sido um importante indicativo de mudanças em seu comportamento interpessoal.

\section{Referências}

Beckert, M. (2002). Relação supervisor-supervisionando e a formação do terapeuta: Contribuições da Psicoterapia Analítico-Funcional (FAP). In H. J. Guilhardi, M. B. B. P. Madi, P. P. Queiroz \& M. C. Scoz (Org.). Sobre o comportamento e cognição: Contribuições para a construção da teoria do comportamento (pp. 245256). São Paulo: ESETec Editores Associados.

Brandão, M. Z. S., \& Silveira, J. M. (2004). Manejo de comportamentos clinicamente elevantes. In C. N. Abreu \& H. J. Guilhardi (Org.). Terapia comportamental e cognitivo-comportamental: Práticas clínicas (pp. 194-204). São Paulo: Roca.

Bush, A. M., Kanter, J. W., Callaghan, G. M., Baruck, D. E., Weeks, C. E., \& Berlin, K. S. (2009). A micro-process analysis of Functional Analytic Psychotherap's mechanism of change. Behavior Therapy, 40(3), 280290. doi: 10.1016/j.beth.2008.07.003

Callaghan, G. M. (2006a). The Functional Idiographic Assessment Template (FIAT) system: For use with interpersonally-based interventions including Functional Analytic Psychotherapy (FAP) and FAP-enhanced treatments. The Behavior Analyst Today, 7, 357-398.

Callaghan, G. M. (2006b). Functional analytic psychotherapy and supervision. International Journal of Behavioral and Consultation Therapy, 2(3), 416-431.

Callaghan, G. M., Follette, W. C., Ruckstuhl, L. E., \& Linnerooth, P. J. N. (2008). The functional analytic psychotherapy rating scale: A behavioral psychotherapy coding system. The Behavior Analyst Today, 9, 98-116.

Callaghan, G. M., Summer, C. J., \& Weidman, M. (2003). The treatment of histrionic and narcissistic personality disorder behaviors: A single-subject demonstration of clinical improvement using functional analytic psychotherapy. Journal of Contemporary Psychotherapy, 33(4), 321-328.
Catania, A. C. (1999). Aprendizagem, pensamento e cognição (4a ed.). Porto Alegre: Artmed.

Conte, F. C. S., \& Brandão, M. Z. S. (2001). Psicoterapia Funcional-Analítica: 0 potencial de análise da relação terapêutica no tratamento de transtornos de ansiedade e de personalidade. In B. Range (Org.). Psicoterapias cognitivo-comportamentais: Um diálogo com a psiquiatria (pp. 19-33). Porto Alegre: Artmed.

Dinsmoor, J. A. (2004). The etymology of basic concepts in the experimental analysis of behavior. Journal of the Experimental Analysis of Behavior, 3(82), 311-316.

Gottman, J. M., \& Rushe, R. H. (1993). The analysis of change: Issues, fallacies, and new ideas. Journal of Consulting and Clinical Psychology, 61(6), 907-910.

Kanter, J. W., Landes, S. J., Busch, A. M., Rush, L. C., Brown, K. R., \& Baruk, D. E. (2006). The effect of contingent reinforcement on target variables in outpatient psychotherapy for depression: A successful and unsuccessful case using functional analytic psychotherapy. Journal of Applied Behavior Analysis, 39(4), 463-467.

Kanter, J. W., Schildcrout, J. S., \& Kohlenberg, R. J. (2005). In vivo process in cognitive therapy for depression: Frequency and benefits. Psychotherapy Research, 15(4), 366-373.

Kohlenberg, R. J., \& Tsai, M. (1991/2001). Functional Analytic Psychotherapy: Creating intense and curative therapeutic relationships. Nova York: Plenum Press.

Shahan, T. A., \& Chase, P. N. (2002). Novelty, stimulus control, and operant variability. The Behavior Analyst, 25(2), 175-190.

Skinner, B. F. (1935). The generic nature of the concepts of stimulus and response. Journal of General Psychology, 12, 40-65.

Skinner, B. F. (1979/2003). Ciência e comportamento humano (11a ed.). São Paulo: M. Fontes. (Originalmente publicado em 1953).

Vandenberghe, L. (1999). Uma abordagem contextual da supervisão clínica. In R. A. Banaco(Org.). Sobre comportamento e cognição, aspectos teóricos, metodológicos e de formação em análise do comportamento e terapia cognitivista (pp. 510-515). Santo André: ESETec Editores Associados. 
Vandenberghe, L. (2008). Culture-sensitive Functional Analytic Psychotherapy. The Behavior Analyst, 31(1), 67-79.
Vandenberghe, L. M. A., \& Ferro, C. L. B. (2005). Terapia de grupo baseada em psicoterapia analítico funcional como abordagem terapêutica para dor crônica. Psicologia, 7(1), 137-152. 